\title{
OPTICAL COHERENCE TOMOGRAPHY ANGIOGRAPHY IN RETINAL VEIN OCCLUSION
}

\section{Correlations Between Macular Vascular Density, Visual Acuity, and Peripheral Nonperfusion Area on Fluorescein Angiography}

DANIEL SEKNAZI, MD, FLORENCE COSCAS, MD, ALEXANDRE SELLAM, MD, FABIEN ROUIMI, MD, GABRIEL COSCAS, MD, ERIC H. SOUIED, MD, PHD, AGNÈS GLACET-BERNARD, MD

Purpose: To study correlations in patients with retinal vein occlusion between the automatically quantified macular vascular densities in the superficial and deep capillary plexus (DCP) obtained using optical coherence tomography angiography (OCTA) and the data from conventional examination, particularly visual acuity and peripheral retinal nonperfusion assessed using fluorescein angiography (FA).

Methods: Retrospective, observational study of patients with retinal vein occlusion who underwent a comprehensive ophthalmic examination including FA and OCTA using the AngioVue OCTA system version 2015.100.0.35 (OptovueRTVue XR 100; AVANTI, Inc, Fremont, CA). Vascular densities in the superficial capillary plexus and DCP, as well as the area of the foveal avascular zone, were measured using the AngioAnalytics software.

Results: Our study of 65 eyes of 61 patients (33 men, mean age: 67 years) showed a significant correlation between peripheral nonperfusion on FA and (1) automatically quantified global vascular density in both plexus $(P=0.021$ for the DCP) and (2) foveal avascular zone area $(P=0.037)$. We also found significant correlations between capillary dropouts in both plexus and peripheral nonperfusion $(P<0.001$ for both) and between visual acuity and vascular densities $(P=0.002$ for the global density in the DCP). Global density less than $46 \%$ in the DCP was associated to the presence of peripheral nonperfusion area on FA $(P=0.003)$ and to enlargement of the superficial foveal avascular zone $(P=0.002)$.

Conclusion: Our study demonstrated a significant correlation between automatically quantified macular vascular density on OCTA and peripheral nonperfusion on FA; OCTA could help identify high-risk retinal vein occlusion patients who may benefit from further evaluation using FA.

RETINA 38:1562-1570, 2018

$\mathrm{R}$ etinal vein occlusion (RVO) is a major cause of vision loss and impairment throughout the world after diabetic retinopathy and its prevalence increases with age. ${ }^{1}$ Retinal ischemia is the main complication of nonperfused forms of RVO and may result in macular ischemia which limits visual recovery and/or in anterior segment neovascularization and neovascular glaucoma. ${ }^{2}$ Since the early 70s, fluorescein angiography (FA) has been the gold standard for detecting and evaluating the presence of retinal perfusion in clinical practice. . $^{3-6}$
Recently, the development of optical coherence tomography angiography (OCTA) $)^{7,8}$ has provided a new noninvasive method for imaging the superficial and deep capillary networks in the posterior pole. This novel technique has been studied in both normal and pathologic eyes, particularly in patients with retinal vascular disorders such as diabetic retinopathy ${ }^{9}$ and RVO. ${ }^{10,11}$ In RVO, capillary dropout was predominantly observed at the level of the deep capillary plexus (DCP), the perifoveal arcade was frequently 
disrupted, and the foveal avascular zone (FAZ) was enlarged. ${ }^{10,12-14}$ Optical coherence tomography angiography was shown to be more effective than FA in RVO to display and analyze the macular capillary network and the perifoveal capillary arcade and also to detect the presence of macular edema. Perifoveal capillary arcade disruption observed using OCTA was found to be correlated with the presence of peripheral nonperfusion on FA. ${ }^{12}$

The new Angioanalytics software in OCTA allows automated measurements of vascular density, in different retinal sectors, at the level of the superficial capillary plexus (SCP) and DCP. Age-related vascular density mapping data, using OCTA in healthy subjects at the level of the SCP and DCP, have been provided to improve the concept of vascular density grading which showed high inter- and intraexaminer repeatability and interexaminer reproducibility. ${ }^{15}$ Vascular density in RVO was demonstrated to be lower than in healthy subjects matched for age and sex ${ }^{10,15,16}$ and in the fellow eyes. ${ }^{10,16,17}$ Optical coherence tomography angiography demonstrated a slight decrease in average macular vascular density, despite the resolution of macular edema, in patients with RVO with macular edema who were treated with intravitreal injections of antivascular endothelial growth factors or with dexamethasone. ${ }^{18,19}$

The aim of this study was to evaluate the correlations in patients with RVO between the automatically quantified macular vascular density and FAZ area obtained using OCTA and the data obtained from conventional examination, particularly visual acuity and peripheral retinal nonperfusion assessed using FA.

\section{Materials and Methods}

This was a retrospective, observational series of patients with RVO. Our study adhered to the tenets of the 1964 Declaration of Helsinki and was approved by the Institutional Review Board of Creteil University, Creteil, France. All study patients provided written informed consent for participation in this study. The case

\footnotetext{
From the Department of Ophthalmology, Centre Hospitalier Intercommunal de Créteil, Université Paris-Est-Créteil, Paris 12 University, Créteil, France.

None of the authors has any financial/conflicting interests to disclose.

This is an open-access article distributed under the terms of the Creative Commons Attribution-Non Commercial-No Derivatives License 4.0 (CCBY-NC-ND), where it is permissible to download and share the work provided it is properly cited. The work cannot be changed in any way or used commercially without permission from the journal.

Reprint requests: Florence Coscas, MD, Department of Ophthalmology, Centre Hospitalier Intercommunal de Créteil, 40 Avenue Verdun, Université Paris Est, 94010 Créteil, France; e-mail: coscas.f@gmail.com
}

records of consecutive patients with RVO, both naive and already treated, who presented to our department from November 1, 2015 to July 31, 2016 were reviewed.

The inclusion criterion was the presence RVO, either central RVO (CRVO), hemicentral RVO, or branch RVO (BRVO). The exclusion criteria were patients with poor-quality images on OCTA (signal strength index lower than 50) because of significant eye movements or lens opacities, previous retinal surgery or laser retinal photocoagulation, pathologic myopia, ocular trauma, and the presence of other retinal disorders such as diabetic retinopathy or agerelated macular degeneration.

The following data were obtained from the case records of our study patients: best-corrected visual acuity, anterior segment examination, intraocular pressure, FA, spectral domain OCT (Spectralis Heidelberg Engineering, Heidelberg, Germany), and OCTA using the AngioVue OCTA system version 2015.100.0.35 (OptovueRTVue XR 100; AVANTI, Inc) intended for retinal imaging (AngioRetina mode, software AngioAnalytics) as previously described. ${ }^{7,20,21}$

DualTrac Motion Correction Technology provides two levels of motion correction. The first one, realtime tracking, is done during acquisition and corrects for large eye movements such as saccades and patient blinking. The second level motion correction technology is performed during postprocessing and is a pixel-level correction of small artifacts. The calculated amplitude decorrelation signal from consecutive B-scans allows blood flow and, therefore, the capillary network to be clearly visualized. Image analysis was performed using automated retinal segmentation derived from the machine software. Motion correction was performed using registration of two orthogonally captured imaging volumes. The scanning area was captured in $3 \times 3$ sections and was centered on the fovea. The SCP (between $3 \mu \mathrm{m}$ below the inner limiting membrane and $15 \mu \mathrm{m}$ below the inner border of the inner plexiform layer) and the DCP (between $15 \mu \mathrm{m}$ below the inner border of the inner plexiform layer and $70 \mu \mathrm{m}$ below the inner border of the inner plexiform layer) were defined as previously published. ${ }^{22}$

Several parameters were used to evaluate the capillary network. First, we used the AngioAnalytics software to calculate the relative density of flow as a percentage of the total area. With AngioAnalytics software, vessel density is calculated by first extracting a binary image of the vessels from the gray scale OCTA en-face image, and then by computing the percentage of pixels of vessels in the defined sectors (for the Early Treatment Diabetic Retinopathy Study flowchart) or "in the entire en-face image based on 
the binary image" (for the vascular map density. The area used for vascular density quantification in this study was the parafoveal area, which corresponds to the macular ring measured between $1 \mathrm{~mm}$ and $2.5 \mathrm{~mm}$ from the center of the fovea. We also analyzed the no-flow areas, using the automated measurement of nonflow, which include the foveal avascular area of the FAZ. Second, qualitative features such as disruption of the FAZ and capillary dropout were recorded at the SCP and the DCP. Capillary dropout on both plexus was graded from 0 to 8 ( 0 being no capillary dropout and eight extensive capillary dropout). The OCTA cartography permitted easily to obtain nine fields: the four quadrants of the parafoveal ring in the ETDRS fields, each split in two by the automatic vertical and the horizontal lines, providing eight parafoveal fields and, in the center, the fovea. Only the eight parafoveal fields were considered for the study purpose (the fovea was excluded). The result of the grading consists of the number of parafoveal fields involved by the disruption of the FAZ or the capillary dropout. All the fields involved with capillary disruption were added up and corresponded to the grade of the capillary dropout evaluation (from 0 to 8 ).

Fluorescein angiography was performed using a $55^{\circ}$ lens, and 8 peripheral fields were taken just after the filling of all retinal vessels (between $30 \mathrm{sec}-$ onds and 90 seconds for most exams) to evaluate peripheral nonperfusion area. One experimented clinician (AGB) graded peripheral nonperfusion on FA from one to six in a blind manner to the OCTA images to prevent bias. Grade 1 corresponded to a wellperfused peripheral retina, Grade 2 to questionable nonperfusion because of staining and leakage of dye from the vessel wall in the peripheral retina or because of retinal hemorrhages, Grade 3 was the presence of moderate nonperfused peripheral area of less than one quadrant, Grade 4 was the presence of marked nonperfused peripheral area larger than Grade 3 and less than two quadrants, Grade 5 was the presence of severe nonperfused peripheral area larger than Grade 4 and less than four quadrants, and finally Grade 6 corresponded to total retinal ischemia including the posterior pole. ${ }^{4,23}$

Best-corrected visual acuity was converted to the logarithm of the minimum angle resolution equivalents before statistical analyses. The Fischer's exact test was used for qualitative variables. Comparisons of medians of independent variables were performed using the Mann-Whitney test. To evaluate the linear correlation between the FA and OCTA parameters, the Pearson correlation coefficient was calculated. A $P$ value $<0.05$ was considered statistically significant. All statistical analyses were performed using IBM SPSS Statistics version 19.0 (Chicago, IL).

\section{Results}

A total of 65 RVO eyes from 61 consecutive patients were included in this study: CRVO (28 eyes, $43 \%$ ), hemicentral RVO (9 eyes, 14\%), and BRVO (28 eyes, 43\%). For statistical purposes, hemicentral RVO and BRVO eyes were analyzed together in the BRVO subgroup. The age of the patients at presentation ranged from 31 to 91 (mean 67 years; SD \pm $20.5)$ with 33 (54\%) male patients. The mean visual acuity at presentation was 20/80 (Table 1 ).

On FA, $12(18 \%)$ eyes had no sign of peripheral nonperfusion (Grade 1), 8 (12\%) had questionable nonperfusion which was not clearly identified on FA (Grade 2), 14 (22\%) had a nonperfusion peripheral area smaller than one quadrant (Grade 3), 17 (26\%) had a nonperfusion area between one and two quadrants (Grade 4), 9 (14\%) had a nonperfusion area between two and four quadrants (Grade 5), and five

Table 1. Demographic and Clinical Data of Patients With RVO

\begin{tabular}{lccc}
\hline & Total Group $(\mathrm{n}=65)$ & CRVO $(\mathrm{n}=28)$ & BRVO and HCVO $(\mathrm{n}=37)$ \\
\hline Age, year & $67.36 \pm 20.5$ & $66.42 \pm 10.6$ & $68.08 \pm 24.04$ \\
Male (\%) & $33(54)$ & $16(57)$ & $17(45)$ \\
BCVA (LogMAR) & 0.54 & 0.57 & 0.53 \\
(Snellen equivalent) & $20 / 80$ & $20 / 63$ & $20 / 80$ \\
Nonperfusion grade on FA: & $12(18 \%)$ & $10(36 \%)$ & $2(5 \%)$ \\
1 (absent) & $8(12 \%)$ & $4(14 \%)$ & $4(11 \%)$ \\
2 (questionnable) & $14(22 \%)$ & $3(11 \%)$ & $11(30 \%)$ \\
3 (<1 quadrant) & $17(26 \%)$ & $2(7 \%)$ & $15(41 \%)$ \\
4 (<2 quadrants) & $9(14 \%)$ & $4(14 \%)$ & $5(13 \%)$ \\
5 (<4 quadrants) & $5(8 \%)$ & $5(18 \%)$ & - \\
\hline
\end{tabular}

HCVO, hemicentral retinal vein occlusion. 
eyes (8\% of the total group; corresponding to $18 \%$ of CRVO) had a totally nonperfused retina.

Both the SCP and DCP were evaluated using OCTA. The mean signal strength of OCTA images was good at 65.4 (SD 6.0). The perifoveal arcade was disrupted in $22(80 \%)$ BRVO eyes and 20 (73\%) CRVO eyes. The qualitative nonperfusion evaluation (graded from 0 to 8 ) averaged $2.16 \pm 1.98$ in the SCP and $2.55 \pm 1.97$ in the DCP. The vascular density results are shown in Table 2. The mean whole en face density was $43.08 \pm 4.88$ in the SCP and $46.81 \pm$ 4.98 in the DCP (total group). The FAZ area was larger in CRVO than in BRVO $(0.768 \pm$ $0.623 \mathrm{~mm}^{2}$ and $0.515 \pm 0.581 \mathrm{~mm}^{2}$, respectively, in the SCP). Figures 1 and 2 show the pictures of two study patients.

Although there was a large dispersion of the measurements of vascular densities in the different grades of peripheral nonperfusion on FA (Figure 3), statistical analyses showed a significant correlation between peripheral nonperfusion on FA and the automatically quantified macular whole en face vascular density in the SCP (Pearson correlation coefficient $(\mathrm{r})=-0.244, P=0.050)$ and $\mathrm{DCP}(\mathrm{r}=-0.287$, $P=0.021)$. Correlations were also found between the FAZ area and peripheral nonperfusion $(\mathrm{r}=$ $0.260, P=0.037$ ) and between the qualitative grading of nonperfusion in both plexus and peripheral nonperfusion on FA $(\mathrm{r}=0.583, P<0.001$ in the $\mathrm{SCP})$. In BRVO, the vascular density in the ETDRS sector of the occlusion (inferior or superior) in the SCP was correlated with the extent of peripheral nonperfusion on FA $(P=0.033)$. However, peripheral nonperfusion was not correlated with either age or visual acuity.

Visual acuity was correlated with several measurements of vascular densities in the SCP $(\mathrm{r}=-0.389$, $P=0.003$ for the whole en face vascular density) and DCP $(\mathrm{r}=-0.399, P=0.002$ for the whole en face vascular density) but not with FAZ area.

Based on our results, we tried to define a threshold value of vascular density on OCTA that could estimate the extent of peripheral nonperfusion on FA. In the CRVO subgroup $(n=28)$, when the whole en face vascular density in the DCP was more than $46 \%$, there was peripheral nonperfusion of one quadrant or more in only 1 of $13(7.7 \%)$ eyes versus 10 of $15(66.6 \%)$ eyes when vascular density was less than $46 \%$. This difference was statistically significant $(P=0.002)$.

In the BRVO subgroup $(n=37)$, when we analyzed only the affected side (superior sector for superior BRVO and inferior sector for inferior BRVO), we found that when the vascular density in the DCP was less than $46 \%$, there was presence of peripheral

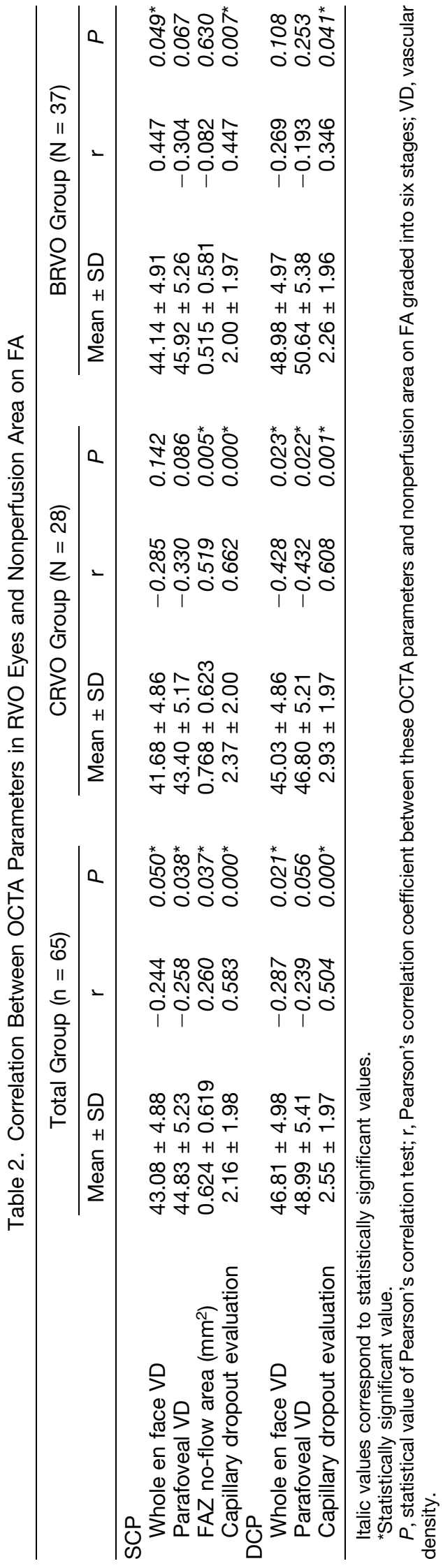



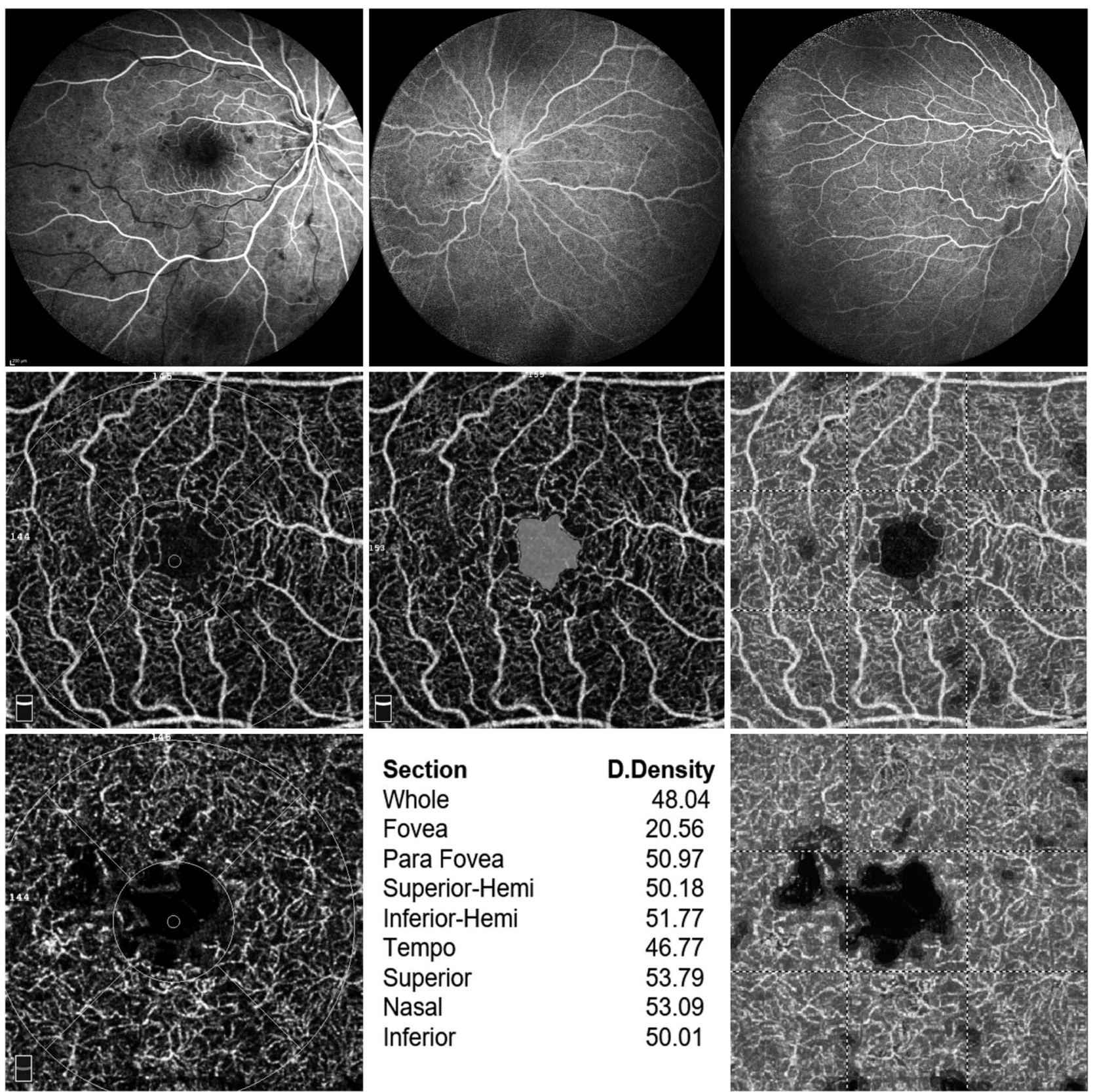

$\begin{array}{lc}\text { Section } & \text { D.Density } \\ \text { Whole } & 48.04 \\ \text { Fovea } & 20.56 \\ \text { Para Fovea } & 50.97 \\ \text { Superior-Hemi } & 50.18 \\ \text { Inferior-Hemi } & 51.77 \\ \text { Tempo } & 46.77 \\ \text { Superior } & 53.79 \\ \text { Nasal } & 53.09 \\ \text { Inferior } & 50.01\end{array}$

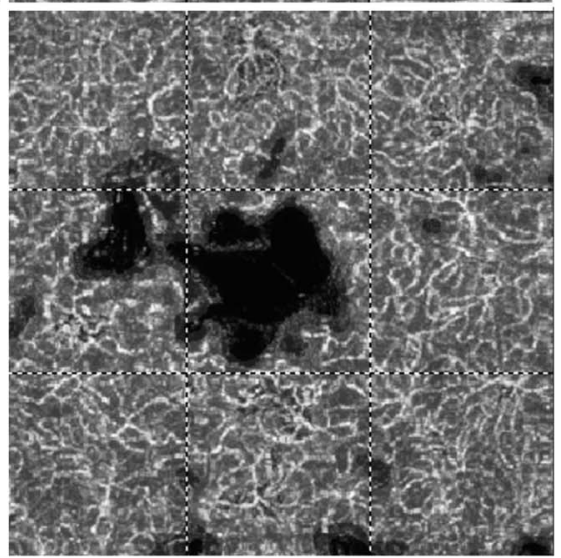

Fig. 1. Multimodal imaging in a 86-year-old patient with CRVO (BCVA: 20/63). One hundred two degree FA (top) shows the well-perfused peripheral retina (Grade 1). On OCTA, at the superficial capillary plexus (middle), few anastomotic arcade disruptions are seen (middle left), virtual color grid of macular densities (middle right) shows subnormal values. Whole en face vascular density in the SCP was 49.65\%. In the deep capillary plexus (bottom left) and the virtual color grid (bottom right), hypoperfused areas are seen. Whole en face vascular density in the DCP was $48.04 \%$. Superficial nonflow area was $0.221 \mathrm{~mm}^{2}$ (middle of the second line).

retinal nonperfusion in $16 / 17$ eyes $(94 \%)$ versus in $15 / 20$ eyes $(75 \%)$ when the vascular density was more than $46 \%(P=0.13)$.

Table 3 shows that the presence of peripheral nonperfusion and the enlargement of the FAZ were statistically correlated with whole en face vascular density in the DCP inferior to $46 \%(P=0.003$ and $P=0.002$, respectively).

\section{Discussion}

This study of 65 RVO eyes has shown, for the first time, a significant correlation between retinal peripheral nonperfusion assessed using FA and the macular vascular densities, in the SCP and DCP, measured using OCTA. The distribution of vascular densities in each grade of peripheral retinal nonperfusion showed 

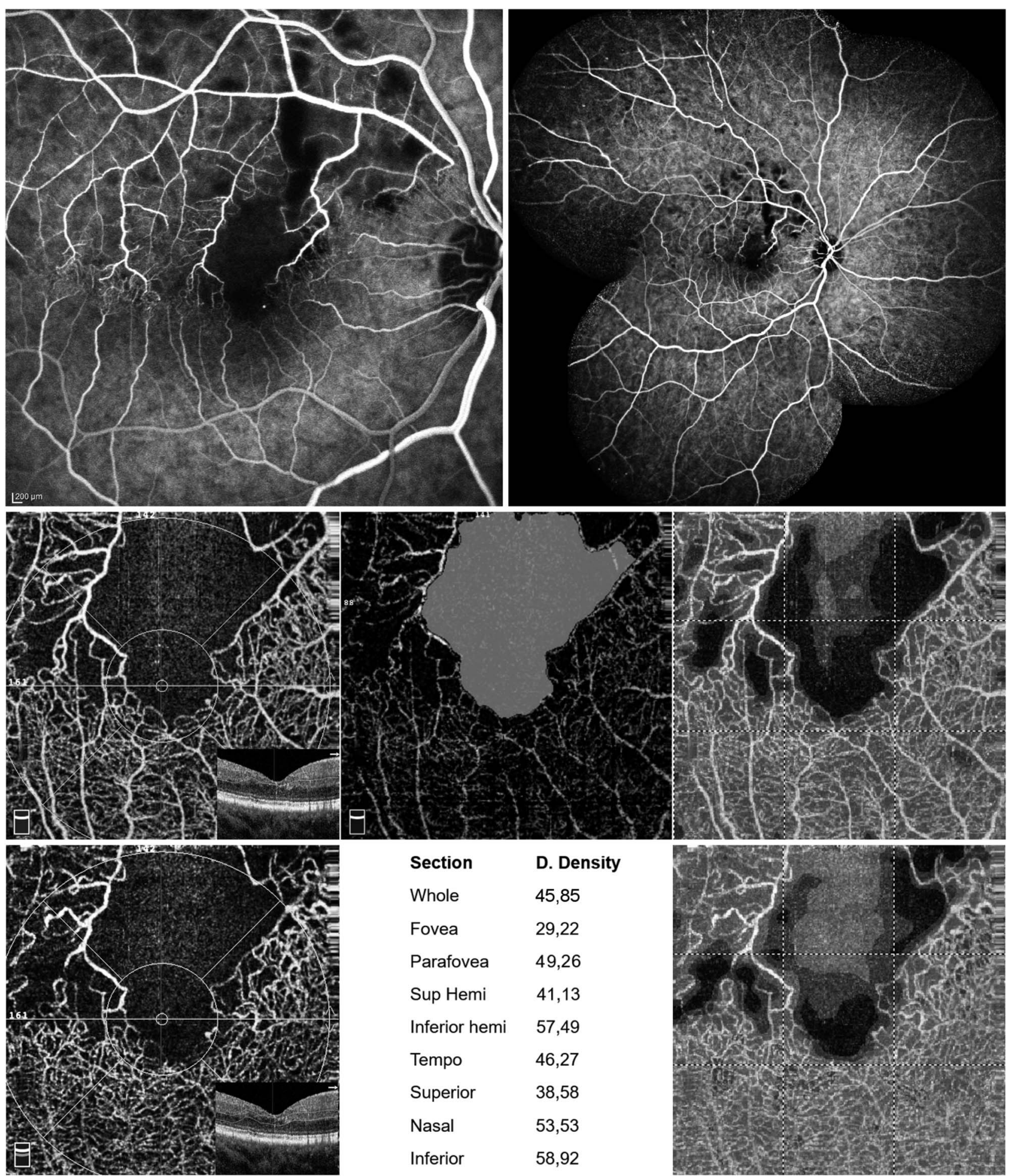

$\begin{array}{ll}\text { Section } & \text { D. Density } \\ \text { Whole } & 45,85 \\ \text { Fovea } & 29,22 \\ \text { Parafovea } & 49,26 \\ \text { Sup Hemi } & 41,13 \\ \text { Inferior hemi } & 57,49 \\ \text { Tempo } & 46,27 \\ \text { Superior } & 38,58 \\ \text { Nasal } & 53,53 \\ \text { Inferior } & 58,92\end{array}$

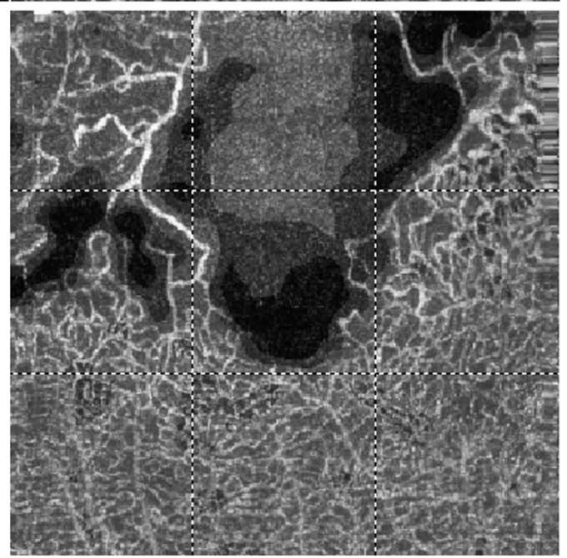

Fig. 2. Multimodal imaging in a 68-year-old patient with BRVO (BCVA: 20/40). Fluorescein angiography (top left) shows the enlargement of the FAZ and macular nonperfusion; on the composite of peripheral fields, the supero-temporal quadrant was nonperfused (Grade 3). On OCTA, at the SCP (middle), numerous capillary dropouts and anastomotic arcade disruptions are seen in the superior sector (middle left), and virtual color grid of macular densities (middle right) shows decreased values in the parafoveal and superior sectors. Whole en face vascular density in the SCP was $41.98 \%$. In the DCP (bottom left) and the virtual color grid (bottom right), hypoperfused areas are seen. Whole en face vascular density in the DCP was $45.95 \%$. Superficial nonflow area was $2,089 \mathrm{~mm}^{2}$ (middle of the second line). 
Fig. 3. Boxplot showing the distribution of vascular density values in the DCP (bottom) according to the grade of peripheral retinal nonperfusion assessed using FA. The first column represents eyes with no or minimal nonperfusion (inferior to one quadrant), and the second column represents eyes with peripheral nonperfusion of one quadrant or more. The central rectangle spans the first quartile to the third quartile (50\% of the values), and the segment inside the rectangle shows the median. The difference in distribution of whole en face vascular density between both subgroups was statistically significant $(P=0.009$ using Mann-Whitney test).

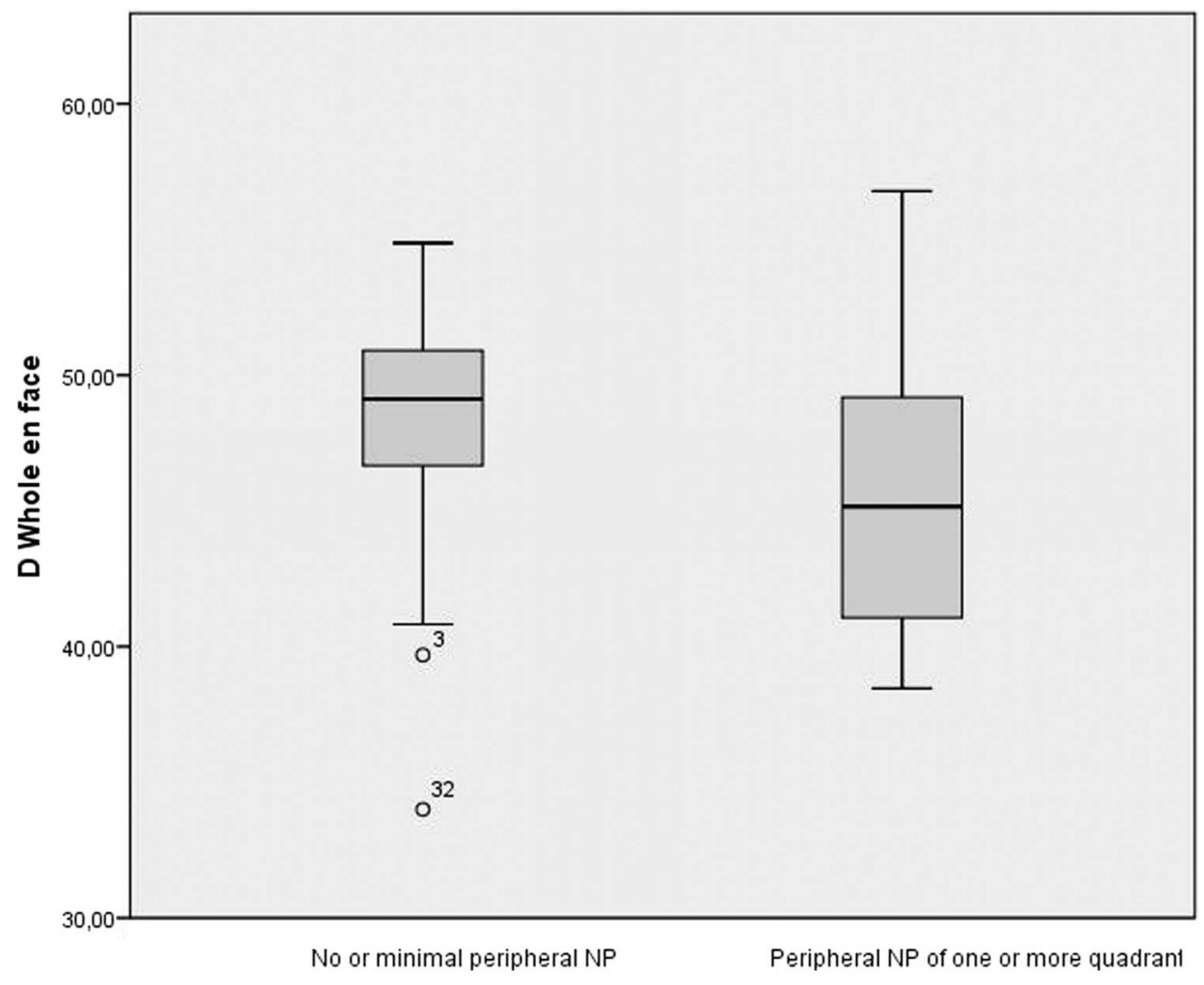

a large dispersion and, therefore, we could not identify a clear threshold for macular vascular density beyond which retinal nonperfusion was always present.

Nevertheless, our study has enabled us to identify eyes with probable significant peripheral retinal nonperfusion that could benefit from FA. In the CRVO subgroup $(n=28)$, when the whole en face vascular density in the DCP was more than $46 \%$, there was peripheral nonperfusion of one quadrant or more in only one of $13(7.7 \%)$ eyes versus 10 of $15(66.6 \%)$ eyes when vascular density was less than $46 \%(P=$ $0.002)$. Therefore, patients with vascular density inferior to $46 \%$ could benefit from FA to detect marked peripheral nonperfusion, which could be responsible for recurrent macular edema or anterior segment neovascularization according to the results of the Central Vein Occlusion Study in 1997.24

In a previous study using the same OCTA device, ${ }^{15}$ we demonstrated that the mean whole en face vascular density in healthy subjects was $52.58 \pm 3.22 \%$ at the level of the SCP and $57.87 \pm 2.82 \%$ at the level of the DCP. Moreover, numerous studies have previously demonstrated that vascular densities are highly decreased in patients with RVO compared with controls or with the fellow eye. ${ }^{16,17}$ Previously, our team compared the vascular density of patients with RVO with the results of healthy controls matched for age and sex, from the normative database ${ }^{15}$ and found out a statistically

Table 3. Comparison of Visual Acuity and FA Peripheral Nonperfusion According to the Value of Whole En Face Vascular Density in the DCP Using OCTA

\begin{tabular}{lccc}
\hline & $\begin{array}{c}\text { Whole En Face } \\
\text { Vascular Density }<46\end{array}$ & $\begin{array}{c}\text { Whole En Face } \\
\text { Vascular Density }>46\end{array}$ & $P$ \\
\hline Visual acuity (LogMAR) & $0.67 \pm 0.41$ & $0.47 \pm 0.42$ & 0.050 \\
Age, year & $69.81 \pm 10.75$ & $65.74 \pm 14.67$ & 0.453 \\
Nonperfusion area on FA & $3.96 \pm 1.66$ & $2.82 \pm 1.25$ & $0.003^{*}$ \\
Superficial FAZ no-flow area, mm ${ }^{2}$ & $0.89 \pm 0.73$ & $0.44 \pm 0.46$ & $0.002^{*}$ \\
Nonperfusion evaluation (SCP) & $3.13 \pm 2.42$ & $1.55 \pm 1.37$ & $0.006^{*}$ \\
Nonperfusion evaluation (DCP) & $3.79 \pm 2.26$ & $1.76 \pm 1.55$ & $0.000^{*}$ \\
\hline
\end{tabular}

${ }^{*}$ Statistically significant value.

$P$, statistical value of Mann-Whitney nonparametric test. 
significant difference: mean whole en face vascular densities reached 50.9 and 56.6 in the SCP and DCP, respectively, in the control group and 46.4 and 48.1 in the RVO group ( $P<0.001$ for both). ${ }^{18,19}$

Our study demonstrated a strong correlation $(P \leq$ 0.001 ) between peripheral nonperfusion on FA and the qualitative evaluation of macular capillary dropout. This is consistent with a previous study that showed a correlation between qualitative evaluation of perifoveal arcade disruption on OCTA and nonperfused areas on FA. ${ }^{13}$

The current study found a correlation between macular capillary nonperfusion, vascular density, and FAZ area consistent with previous OCTA studies. ${ }^{14,25,26}$ This study also demonstrated a correlation between FAZ area and peripheral nonperfusion in the $\operatorname{SCP}(P=0.037)$.

We found that visual acuity was correlated with vascular densities in the SCP and DCP. It is easily understandable that vascular densities, which quantitatively measure the degree of macular ischemia, would be correlated with visual function. Such correlation was recently reported by Samara in a study of 17 BRVO eyes and by Kang in 33 eyes with CRVO and BRVO. ${ }^{16,17}$ However, unlike these studies, we did not find a correlation between the FAZ area and visual function. A study by Casselholm de Salles et $\mathrm{al}^{27}$ on 24 patients with CRVO showed a correlation between the superficial FAZ area and visual acuity but not between the deep FAZ area and visual acuity. This discrepancy may be due to the small size of the study sample.

Our observations in patients with RVO seem to be very similar to those published about the ischemic component of diabetic maculopathy. ${ }^{28}$ The FAZ area had a strong positive correlation with the degree of capillary nonperfusion in diabetic retinopathies, ${ }^{29}$ whereas changes in capillary density and morphology were significantly correlated with worsening diabetic retinopathy and macular edema. ${ }^{30}$ Similarly, we found that low vascular density was correlated with the ischemic form of RVO.

In our retrospective study, we had used FA with a $55^{\circ}$ lens to assess, with peripheral pictures, peripheral retinal nonperfusion which was then qualitatively graded into 6 groups. Now, ultrawide field angiographs can explore, in a single frame, $102^{\circ}$ or $200^{\circ}$ of the retina (Heidelberg, Germany or Optos, United Kingdom). New software is being developed to quantify retinal peripheral nonperfusion after correcting distortions in size and shape at the retinal periphery. An ischemic index can be calculated, and it has been shown to be correlated with neovascularization when more than $45 \% .^{31,32}$ Interestingly, this index is similar to the previous ischemic index of $50 \%$ calculated by
Magargal in 1982 using conventional angiographs. ${ }^{33}$ In the current study, ultrawide field FA and quantification of peripheral nonperfusion would have given a more accurate measurement of retinal nonperfusion, and correlations between peripheral nonperfusion and vascular densities would possibly have been stronger. Therefore, further studies in RVO using ultrawide field FA and OCTA are recommended.

Another limit of this study is the heterogeneity of our study patients including naive patients and long-standing RVO, and eyes with and without macular edema. Among our patients, 29/65 (45\%) had a macular edema, and 36/65 (55\%) did not have any macular edema. The automated segmentation was used to be more reproducible, even though there can be unavoidable mistakes in the segmentation. Also, some parameters may alter the accuracy of vascular density measurement in the DCP: no-flow areas may be located along with cystoid spaces and hence cannot be identified by the Angioanalytics software. ${ }^{34}$ Further, partial superimposition of SCP and DCP may overestimate DCP values. ${ }^{8}$ Recent publications related to the fractal dimensional analysis of OCTA have suggested that such techniques may improve the accuracy of vascular density measurement. These advances promise the possibility of determining cutoffs for disease severity with consequent risk stratification and development of optimal management strategies including appropriate monitoring of treatment response. ${ }^{35}$

\section{Conclusion}

For the first time, a significant correlation between peripheral retinal nonperfusion on FA and automatically quantified macular vascular density measured using OCTA has been demonstrated. Also, the FAZ area was significantly correlated with peripheral nonperfusion on FA and vascular density on OCTA. Therefore, OCTA may help identify eyes with low vascular densities which are at high risk of neovascular complications and could benefit from FA. Further studies using ultrawide field angiography quantification of retinal peripheral nonperfusion and its correlation with vascular density measured using OCTA during the natural course of retinal vascular diseases and after treatment are recommended.

Key words: retinal vein occlusion, optical coherence tomography angiography, ischemia, vascular density.

\section{References}

1. Rogers S, McIntosh RL, Cheung N, et al. The prevalence of retinal vein occlusion: pooled data from population studies 
from the United States, Europe, Asia, and Australia. Ophthalmology 2010;117:313-319.e1.

2. Hayreh SS, Zimmerman MB. Fundus changes in central retinal vein occlusion. Retina 2015;35:29-42.

3. Mendis KR, Balaratnasingam C, Yu P, et al. Correlation of histologic and clinical images to determine the diagnostic value of fluorescein angiography for studying retinal capillary detail. Invest Ophthalmol Vis Sci 2010;51:5864-5869.

4. Glacet-Bernard A, Coscas G, Chabanel A, et al. Prognostic factors for retinal vein occlusion: prospective study of 175 cases. Ophthalmology 1996;103:551-560.

5. Coscas G, Cunha-Vaz J, Soubrane G. Macular edema: definition and basic concepts. Dev Ophthalmol 2010;47:1-9.

6. Jonas J, Paques M, Mones J, Glacet-Bernard A. Retinal vein occlusions. Dev Ophthalmol 2010;47:111-135.

7. Spaide RF, Klancnik JM Jr, Cooney MJ. Retinal vascular layers imaged by fluorescein angiography and optical coherence tomography angiography. JAMA Ophthalmol 2015;133:45-50.

8. Bonnin S, Mané V, Couturier A, et al. New insight into the macular deep vascular plexus imaged by optical coherence tomography angiography. Retina 2015;35:2347-2352.

9. De Carlo TE, Chin AT, Joseph T, et al. Distinguishing diabetic macular edema from capillary nonperfusion using optical coherence tomography angiography. Ophthalmic Surg Lasers Imaging Retina 2016;47:108-114.

10. Adhi M, Filho MA, Louzada RN, et al. Retinal capillary network and foveal avascular zone in eyes with vein occlusion and fellow eyes analyzed with optical coherence tomography angiography. Invest Ophthalmol Vis Sci 2016;57:OCT486-OCT494.

11. Suzuki N, Hirano Y, Yoshida M, et al. Microvascular abnormalities on optical coherence tomography angiography in macular edema associated with branch retinal vein occlusion. Am J Ophthalmol 2016;161:126-132.

12. Kuehlewein L, An L, Durbin MK, Sadda SR. Imaging areas of retinal nonperfusion in ischemic branch retinal vein occlusion with swept-source OCT microangiography. Ophthalmic Surg Lasers Imaging Retina 2015;46:249-252.

13. Coscas F, Glacet-Bernard A, Miere A, et al. OCT-angiography in retinal vein occlusion: evaluation of superficial and deep capillary plexa. Am J Ophthalmol 2016;161:160-171.

14. Kashani AH, Lee SY, Moshfeghi A, et al. Optical coherence tomography angiography of retinal venous occlusion. Retina 2015;35:2323-2331.

15. Coscas F, Sellam A, Glacet-Bernard A, et al. Normative data for vascular density in superficial and deep capillary plexus of healthy adults assessed by optical coherence tomography angiography. Invest Ophthalmol Vis Sci 2016;57:211-223.

16. Kang JW, Yoo R, Jo YH, Kim HC. Correlation of microvascular structures on optical coherence tomography angiography with visual acuity in retinal vein occlusion. Retina 2016. Epub ahead of print.

17. Samara WA, Shahlaee A, Sridhar J, et al. Quantitative optical coherence tomography angiography features and visual function in eyes with branch retinal vein occlusion. Am J Ophthalmol 2016;166:76-83.

18. Sellam A, Glacet-Bernard A, Coscas F, et al Using optical coherence tomography angiography of retinal vein occlusion treated with anti-VEGF. Retina 2016. Epub ahead of print.
19. Glacet-Bernard A, Sellam A, Coscas F, et al. Optical coherence tomography angiography in retinal vein occlusion treated with dexamethasone implant: a new test for follow-up evaluation. Eur J Ophthalmol 2016;26:460-468.

20. Jia Y, Tan O, Tokayer J, et al. Split-spectrum amplitudedecorrelation angiography with optical coherence tomography. Opt Express 2012;20:4710-4725.

21. Gao SS, Jia Y, Zhang M, et al. Optical coherence tomography angiography. Invest Ophthalmol Vis Sci 2016;57:27-36.

22. Matsunaga D, Yi J, Puliafito CA, Kashani AH. OCT angiography in healthy human subjects. Ophthalmic Surg Lasers Imaging Retina 2014;45:510-515.

23. A randomized clinical trial of early pan retinal photocoagulation for ischemic central vein occlusion. The Central Vein Occlusion Study Group N report. Ophthalmology 1995;102: 1434-1444.

24. The Central Vein Occlusion Study Group. Natural history and clinical management of central retinal vein occlusion. Arch Ophthalmol 1997;115:486-491.

25. Nobre Cardoso J, Keane PA, Sim DA, et al. Systematic evaluation of optical coherence tomography angiography in retinal vein occlusion. Am J Ophthalmol 2016;163:93-107.e6.

26. Balaratnasingam $\mathrm{C}$, Inoue $\mathrm{M}$, Ahn $\mathrm{S}$, et al. Visual acuity is correlated with the area of the foveal avascular zone in diabetic retinopathy and retinal vein occlusion. Ophthalmology 2016; 123:2352-2367.

27. Casselholm de Salles M, Kvanta A, Amrén U. Epstein D Optical coherence tomography angiography in central retinal vein occlusion: correlation between the foveal avascular zone and visual acuity. Invest Ophthalmol Vis Sci 2016;57:242-246.

28. Agemy SA, Scripsema NK, Shah CM, et al. Retinal vascular perfusion density mapping using optical coherence tomography angiography in normals and diabetic retinopathy patients. Retina 2015;35:2353-2363.

29. Bresnick GH, Condit R, Syrjala S, et al. Abnormalities of the foveal avascular zone in diabetic retinopathy. Arch Ophthalmol 1984;102:1286-1293.

30. Kim AY, Chu Z, Shahidzadeh A, et al. Quantifying microvascular density and morphology in diabetic retinopathy using spectral-domain optical coherence tomography angiography. InvestOphthalmoVisSci 2016;57:362-370.

31. Tsui I, Kaines A, Havunjian MA, et al. Ischemic index and neovascularization in central retinal vein occlusion. Retina 2011;31:105-110.

32. Tan CS, Chew MC, van Hemert J, et al. Measuring the precise area of peripheral retinal non-perfusion using ultra-widefield imaging and its correlation with the ischaemic index. $\mathrm{Br} \mathrm{J}$ Ophthalmol 2016;100:235-239.

33. Magargal LE, Donoso LA, Sanborn GE. Retinal ischemia and risk of neovascularization following central retinal vein obstruction. Ophthalmology 1982;89:1241-1245.

34. Spaide RF. Volume-rendered optical coherence tomography of retinal vein occlusion pilot study. Am J Ophthalmol 2016;165: 133-144.

35. Zahid S, Dolz-Marco R, Freund KB, et al. Fractal dimensional analysis of optical coherence tomography angiography in eyes with diabetic retinopathy. Invest Ophthalmol Vis Sci 2016;57: 4940-4947. 